\title{
Expressive map design: OGC SLD/SE++ extension for expressive map styles
}

\author{
Sidonie Christophe ${ }^{\mathrm{a}}$, Bertrand Duménieu ${ }^{\mathrm{a}}$, Antoine Masse ${ }^{\mathrm{a}}$, Charlotte Hoarau ${ }^{\mathrm{a}}$, Jérémie Ory ${ }^{\mathrm{a}}$, \\ Mathieu Brédif ${ }^{\mathrm{a}}$, François Lecordix ${ }^{\mathrm{a}}$, Nicolas Mellado ${ }^{\mathrm{b}}$, Jérémie Turbet ${ }^{\mathrm{b}}$, Hugo Loi ${ }^{\mathrm{c}}$, Thomas \\ Hurtut $^{\mathrm{d}}$, David Vanderhaeghe ${ }^{\mathrm{b}}$, Romain Vergne ${ }^{\mathrm{c}}$ and Joëlle Thollot ${ }^{\mathrm{c}}$ \\ ${ }^{a}$ Univ. Paris-Est, LASTIG, IGN, ENSG, F-94160 Saint-Mande, France; firstname.surname@ign.fr \\ ${ }^{b}$ IRIT, Université de Toulouse, CNRS, INPT, UPS, UT1C, UT2J, France; firstname.surname@irit.fr \\ ${ }^{c}$ Univ. Grenoble Alpes, CNRS, Inria; firstname.surname@inria.fr \\ ${ }^{d}$ Polytechnique Montréal, Canada; thomas.hurtut@polymtl.ca
}

\begin{abstract}
In the context of custom map design, handling more artistic and expressive tools has been identified as a carto-graphic need, in order to design stylized and expressive maps. Based on previous works on style formalization, an approach for specifying the map style has been proposed and experimented for particular use cases. A first step deals with the analysis of inspiration sources, in order to extract 'what does make the style of the source', i.e. the salient visual characteristics to be automatically reproduced (textures, spatial arrangements, linear stylization, etc.). In a second step, in order to mimic and generate those visual characteristics, existing and innovative rendering techniques have been implemented in our GIS engine, thus extending the capabilities to generate expressive renderings. Therefore, an extension of the existing cartographic pipeline has been proposed based on the following aspects: 1- extension of the symbolization specifications OGC SLD/SE in order to provide a formalism to specify and reference expressive rendering methods; 2 - separate the specification of each rendering method and its parameterization, as metadata. The main contribution has been described in (Christophe et al. 2016). In this paper, we focus firstly on the extension of the cartographic pipeline (SLD++ and metadata) and secondly on map design capabilities which have been experimented on various topographic styles: old cartographic styles (Cassini), artistic styles (watercolor, impressionism, Japanese print), hybrid topographic styles (ortho-imagery \& vector data) and finally abstract and photo-realist styles for the geovisualization of costal area. The genericity and interoperability of our approach are promising and have already been tested for $3 \mathrm{D}$ visualization.
\end{abstract}

Keywords: map design, geovisualization, map style, expressive rendering, SLD

\section{Introduction}

In the context of custom cartographic representation with geovisualization tools, some issues are still at stake, as abstraction levels and visual variables are still difficult to select to fit users' needs and preferences. Furthermore, some geographic spatio-temporal phenomena are still hard to represent in an understandable way.

A way of personalizing a map is to draw inspiration from existing artistic styles. In practice, this is complex to achieve in a GIS because there is currently no formal way to describe an artistic (complex) style. Rendering techniques are more and more used in map design, to manage photo- or non-photo-realistic rendering and pseudo-natural effects or to mimic artistic and old practices in cartography (Patterson et al. 2004; Trapp et al. 2011; Jenny \& Jenny 2012; Semmo et al. 2013; amongst others). However, there techniques cannot be easily used and con-trolled within a GIS to reproduce a given style.

We address the issue of the specification of 'what the style of a map is', based on previous works on style formalization, both in expressive rendering (Grabli et al. 2004, Willats \& Durand 2005, amongst others) and in map design (Kent \& Vujakovic 2009, Beconyte 2011, Christophe 2012, amongst others). Our main contribution has been published in (Christophe et al. 2016), we focus here on how the extending style formalization in map design allows, in a generic and interoperable way, to design various topographic styles.

In a first step, cartographic needs and design rules have been explored, based on both interviews with cartographers (mainly producers for current map design, and "artist-cartographers" for hand-drawing map design at the time), and the analysis of map design specifications (generalization and legend specification), in order to extract expected salient visual characteristics of "what the style of a map' could be. These requirements are described in the context of several expected styles: current topographic French and Swiss maps (Ory et al. 2015), mountain (by hand) maps, Cassini maps, impressionism and watercolor styles (Christophe et al. 2016). Then, expressive rendering techniques are pickedup from expressive rendering area, and implemented in GIS tools, in order to be able to re-produce or mimic these visual characteristics of map styles. Among these techniques, existing methods for surface filling and line stylization using raster textures have been investigated (Christophe et al. 2016) and an innovative method for 
vectorial texture generation has been proposed (Loi et al. 2013, Loi 2015).

Another important issue is the existing standard formalisms available to manage the map style: in GIS, we mainly rely on the OGC Symbology Encoding (SE) which provides stylization blocks, related to each type of geometry (Müller 2006). And cartographic data are structured accordingly to the OGC Styled Layer Descriptor specification (SLD) (Lupp 2007). These specifications are efficient to specify a typical GIS rendering but not sufficient to man-age expressive rendering techniques and related style specifications with a GIS engine. In order to split the style specification, the rendering techniques specification and implementation levels, we thus propose:

- to add metadata composed of expressive method descriptors that can be called through the extended SE specifications;

- to extend the SE specifications by adding new controllable expressive elements

(ExpressiveStroke and Expressive Fill) which can refer to the name of an expressive method descriptor and describe its set of parameters, defined in the previous metadata.

The GIS engine capabilities have been extended based on the use of OpenGL as a rendering engine. The rendering methods have been implemented using GLSL shaders programs as text parameters and GLSL uniforms for parameter values.
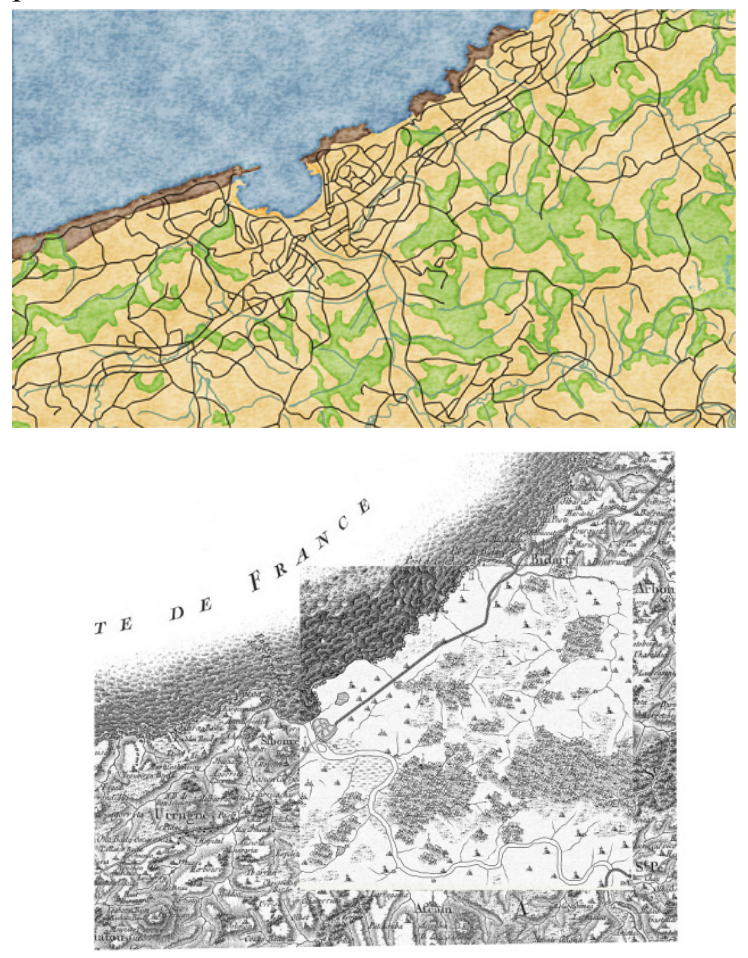

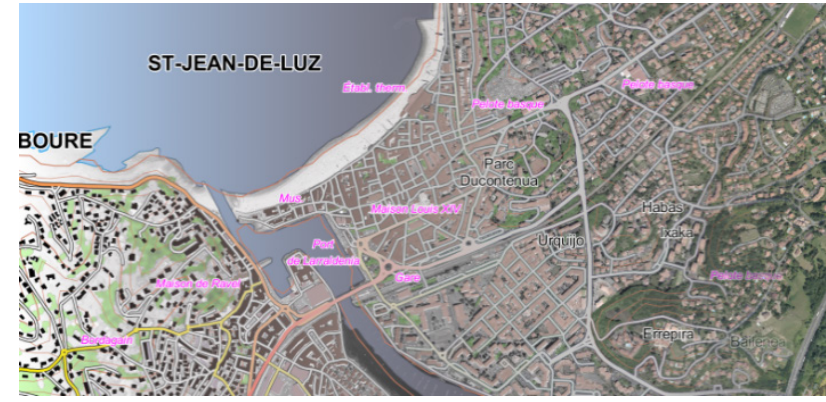

Fig. 1. Various map styles: watercolor, Cassini (Christophe et al. 2016), and ortho-imagery/vector hybrid (Hoarau \& Christophe 2016).

This extension and adaptation of the traditional cartographic pipeline, based on the split of the style specification, the rendering techniques specification and their implementation, has been experimented to generate various map styles such as: old cartographic styles (Cassini), artistic styles, i.e. watercolor, impressionism, or Japanese print in Figure 1 (Christophe et al. 2016), hybrid topographic styles (ortho-imagery \& vector data) (Hoarau \& Christophe 2016) and finally abstract and photo-realist styles for the geovisualization of costal area (Masse \& Christophe 2016). The system flexibility is ensured by the access to the parametrization of styles and rendering techniques, at different levels of difficulty and thus expertise. Style specification and stylization techniques have then been experimented for 3D Visualization, validating our proposition (Brasebin et al. 2016). Further works will provide users interfaces in order to facilitate the map style control by various users with various potential uses.

\section{Acknowledgements}

This work was supported by a grant overseen by the French National Research Agency (ANR) as part of the MapStyle project [ANR-12-CORD-0025].

\section{References}

Beconyte, G. 2011. Cartographic styles: criteria and parameters. In 25th International Cartographic Conference. 3-8 July 2011, Paris, France.

Brasebin M., Christophe S., Jacquinod F., Vinesse A., Mahon H. 3D geovisualization and stylization to manage comprehensive and participative local urban plans, ISPRS Ann. Photogramm. Remote Sens. Spatial Inf. Sci., IV-2-W1, 83-91, doi:10.5194/isprs-annals-IV2-W1-83-2016, 2016

Christophe S., Duménieu B., Turbet J., Hoarau C., Mellado N., Ory J., Loi H., Masse A., Arbelot B., Vergne R., Brédif M., Hurtut T., Thollot J., Vanderhaeghe D.. Map Style Formalization: Rendering Techniques Extension for Cartography. Pierre Bénard; Holger Winnemöller. Expressive 2016 The Joint Symposium on Computational Aesthetics and SketchBased Interfaces and Modeling and Non-Photorealistic Animation and Rendering, May 2016, Lisbonne, Portugal. The Eurographics Association, NonPhotorealistic Animation and Rendering, 2016, NonPhotorealistic Animation and Rendering. 
$<$ http://expressive.richardt.name/2016/>.

$<10.2312 /$ exp.20161064>. $<$ hal-01317403>

Grabli, S., Turquin, E., Durand, F., and Sillion, F. 2004.

Programmable style for NPR line drawing. Rendering Techniques 2004 (Eurographics Symposium on Rendering). ACM Press

Hoarau C., Christophe S. 2016. Cartographic continuum rendering based on color and texture interpolation to enhance photo-realism perception. ISPRS Journal of Photogrammetry and Remote Sensing (in press). DOI:10.1016/j.isprsjprs.2016.09.012

Jenny, H., Jenny, B., 2012. Recycling Cartographic Masterworks - Challenges in Adapting Example-based Texture Synthesis for Panoramic Map Creation. In: Proc. of AutoCarto2012 Conference, Columbus, Ohio, USA, September 16-18, 2012.

Kent, A. and Vujakovic, P. 2009. Stylistic diversity in european state 1 : 50000 topographic maps. Cartographic Journal, The, 46(3):179-213.

Loi, H. Programmable Synthesis of Element Textures and Application to Cartography. Graphics. Université de Grenoble, 2015. English.

Loi, H., Hurtut T., Vergne R., Thollot J. 2013. Discrete Texture Design Using a Programmable Approach, SIGGRAPH Talks, 2013.

Lupp M. 2007. Styled layer descriptor implementation specification. Open Geospatial Consortium Document Number: OGC 05-078r4, Version: 1.1.0 (jun 2007). URL: http://www.opengeospatial.org/standards/sld.

Masse A., Christophe S. 2016. Améliorer la perception $\mathrm{du}$ réalisme dans la géovisualisation du littoral utilisation de données spatio-temporelles hétérogènes. Revue Internationale de Géomatique (RIG) vol.26, n.4 (4-2016), pp 403-424 http://dx.doi.org/10.3166/rig.2016.00008.

Müller M. 2006. Symbology Encoding Implementation Specification, version 1.1.0, OpenGIS Implementation Specification, 05-077r4, OpenGIS Consortium (jul 2006). http://www.opengeospatial.org/standards/symbol.

Ory, J., S. Christophe, S.I. Fabrikant and B. Bucher. 2015. How do map readers recognize a topographic mapping style?, The Carto-graphic Journal. 52(2):193203, May 2015. DOI:10.1080/00087041.2015.1119459.

Patterson, T., Kelso, N. V., Mar. 2004. Hal Shelton Revisited: Designing and Producing Natural-Color Maps with Satellite Land Cover Data. Cartographic Perspectives (47), 28-55.

Semmo, A., Kyprianidis, J. E., Trapp, M., Döllner, J., 2013. Real-Time Rendering of Water Surfaces with Cartography-Oriented De-sign. Proc. of International Symposium on Computational Aesthetics in Graphics, Visualization, and Imaging (CAe), Anaheim, California, USA. pp. 5-14.

Trapp, M., Beesk, C., Pasewaldt, S., Döllner, J., 2011. Interactive rendering techniques for highlighting in $3 \mathrm{~d}$ geovirtual environments. In: Kolbe, T. H., Knig, G.,
Nagel, C. (Eds.), Advances in 3D Geo-Information Sciences. Lecture Notes in Geoinformation and Cartography. Springer Berlin Heidelberg, pp. 197-210.

Willats, J. and Durand, F. (2005). Defining pictorial style: Lessons from linguistics and computer graphics. Axiomathes, 15(3). 